

\title{
Novel imaging techniques in staging oesophageal cancer
}

\author{
Kieran Foley ${ }^{1}$, John Findlay ${ }^{2,3}$ and Vicky Goh ${ }^{4,5}$
}

1. Division of Cancer \& Genetics, School of Medicine, Cardiff University, UK

2. Oxford OesophagoGastric Centre, Churchill Hospital, Oxford University Hospitals NHS Foundation Trust, UK

3. Department of Oncology, Old Road Campus Research Building, Roosevelt Drive, University of Oxford, UK

4. Department of Radiology, Guy's \& St Thomas' NHS Foundation Trust, London, UK

5. School of Biomedical Engineering and Imaging Sciences, King's College London, UK

Corresponding author:

Dr Kieran Foley, Division of Cancer \& Genetics, School of Medicine, Cardiff University, UK

E-mail: foleykg@cardiff.ac.uk

Co-author email addresses:

John Findlay (Oxford) john.findlay@oncology.ox.ac.uk

Vicky Goh (KCL \& GSTT) vicky.goh@kcl.ac.uk

Word Count: 6,978 (including references, practice points and research agenda)

No funding was received for this work. The authors have no conflicts of interest or any financial disclosures to declare. 


\begin{abstract}
The survival of oesophageal cancer is poor as most patients present with advanced disease. Radiological staging of oesophageal cancer is complex but is fundamental to clinical management. Accurate staging investigations are vitally important to guide treatment decisions and optimise patient outcomes. A combination of baseline computed tomography (CT), endoscopic ultrasound (EUS) and positron emission tomography (PET) are currently used for initial treatment decisions. The potential value of these imaging modalities to restage disease, monitor response and alter treatment is currently being investigated. This review presents an essential update on the accuracy of oesophageal cancer staging investigations, their use in re-staging after neo-adjuvant therapy and introduces evolving imaging techniques, including novel biomarkers that have clinical potential in oesophageal cancer.
\end{abstract}

Keywords: Oesophageal cancer; TNM; staging; response; prognosis; computed tomography; endoscopic ultrasound; positron-emission tomography; magnetic resonance imaging 


\section{Novel imaging techniques in staging oesophageal cancer}

Radiological staging is vitally important in oesophageal cancer, serving to define the extent of disease. Accurate staging, along with clinical, pathological and physiological factors, assists clinicians with critical treatment decisions, provides information about a patient's likely prognosis and can stratify patients into risk groups. [1]

\section{Diagnosis and investigation}

Oesophageal cancer is usually diagnosed in symptomatic patients following upper gastrointestinal endoscopy and biopsy. Occasionally, oesophageal tumours are initially diagnosed using computed tomography (CT) in patients suspected of having cancer, or less frequently as an incidental finding. Luminal contrast studies e.g. barium swallow are now rarely performed as a first line investigation, but are useful for assessing the degree of luminal stenosis and guiding oesophageal stent placement. (Fig. 1)

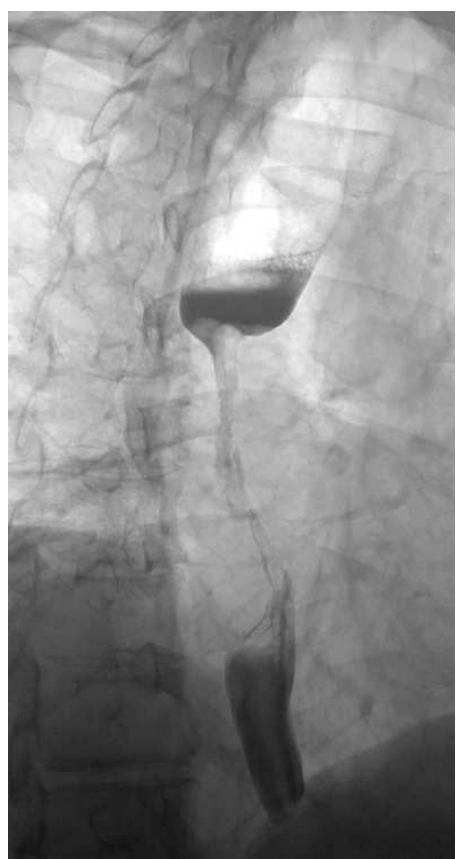


Figure 1. Selected image from a double contrast barium swallow examination showing a stricturing mid oesophageal tumour with proximal retention of oral contrast.

\section{Histology and tumour location}

The two main histological cell types are adenocarcinoma and squamous cell carcinoma (SCC). Adenocarcinomas tend to involve the distal third of the oesophagus and the gastrooesophageal junction (GOJ). SCCS are usually located in the upper and middle thirds of the oesophagus. It is important to define the tumour location and confirm the histological cell type with biopsy because treatments differ accordingly.

TNM staging classification

The globally recognised standard for staging oesophageal and GOJ tumours is the Union for International Cancer Control (UICC) Tumour Node Metastasis (TNM) classification, currently in its $8^{\text {th }}$ edition. [2] (Table 1$)$

Table 1. Tumour Node Metastasis (TNM) $8^{\text {th }}$ edition of the oesophageal cancer staging classification

\begin{tabular}{ll}
\hline Primary tumour $(\mathrm{T})$ \\
\hline TX & Primary tumour cannot be assessed \\
T0 & No evidence of primary tumour \\
Tis & Carcinoma in situ \\
T1 & Tumour invades the lamina propria, muscularis mucosae, or \\
& submucosa \\
T1a & Tumour invades the lamina propria or muscularis mucosae \\
T1b & Tumour invades the submucosa \\
T2 & Tumour invades muscularis propria
\end{tabular}


T3

T4

$\mathrm{T} 4 \mathrm{a}$

$\mathrm{T} 4 \mathrm{~b}$

Tumour invades adventitia

Tumour invades adjacent structures

Tumour invades the pleura, pericardium, azygos vein, diaphragm or peritoneum

Tumour invades other adjacent structures, such as the aorta, vertebral body or trachea

\begin{tabular}{ll}
\hline Regional lymph nodes $(\mathrm{N})$ \\
\hline $\mathrm{NX}$ & Lymph node status cannot be assessed \\
N0 & No regional lymph node metastases \\
N1 & Metastasis in 1-2 regional lymph nodes \\
N2 & Metastasis in 3-6 regional lymph nodes \\
N3 & Metastasis in 7 or more regional lymph nodes \\
& \\
\hline Distant metastases (M) \\
\hline MX & Presence of distant metastases cannot be assessed \\
M0 & No distant metastases \\
M1 & Distant metastases \\
\end{tabular}

One important difference between the $7^{\text {th }}$ and $8^{\text {th }}$ editions are the re-classification of stage sub-categories. The $7^{\text {th }}$ edition stage IIIB and IIIC groups (T3-4a N1-3) are now re-classified as stage IVA, with M1 disease classified as stage IVB. These patients arguably have as poor an outcome as those with distant metastatic disease due to the substantial lymph node burden, which is known to be a major prognostic indicator. [3] Another change to the TNM $8^{\text {th }}$ edition is the separation of T1 tumours into T1a and T1b tumours. This differentiation is important because the risk of lymph node metastases increases from 3-6\% for T1a mucosal tumours, to $21-24 \%$ for T1b submucosal tumours. [4] This differentiation of T1 tumours is important for guiding endoscopic versus surgical management decisions. 
Initial staging with contrast-enhanced CT of the thorax and abdomen +/- pelvis should be performed to define irresectable disease or identify distant metastases that preclude radical therapy. [5] The pelvis should be included in the CT protocol if the primary tumour extends beneath the diaphragm. If the patient is thought to have potentially curable disease, with either surgical or oncological management, then positron emission tomography (PET) combined with CT (PET/CT) is recommended, followed by endoscopic ultrasound (EUS). Diagnostic laparoscopy should also be performed in patients with a tumour involving the stomach. The recently published National Institute for Health and Care Excellence (NICE) guidelines recommends PET/CT for all patients with potentially curable disease (except in patients with T1a tumours) and to only offer EUS in cases where it may change management. [1]

The optimal order of staging investigations is debatable, although it is generally agreed that PET/CT should be performed before EUS. Distant incurable disease detected on PET/CT would prevent a patient from undergoing a potentially risky and unnecessary endoscopic procedure. One study attempted to calculate the most favourable staging strategy using data from 216 consecutive patients. [6] Using likelihood ratios for 4 different staging combinations, the optimal staging strategy was found to be an initial PET/CT, with CT and EUS adding little additional information afterwards. However, the cost-effectiveness of such a strategy must be considered. [7]

The role of EUS is more controversial. A large single-centre study investigated the value of EUS in the staging pathway and concluded that its benefit in tumours staged T2-T4a was minimal compared to potential complications. [8] In contrast, EUS has been found to impact 
on treatment decisions in $29 \%$ of patients, mainly when diagnosing lymph node metastases and when defining the gross tumour volume (GTV) during radiotherapy planning. [9] PET tumour and disease length measurements have been shown to significantly differ, with PET tending to yield shorter dimensions. [10] Furthermore, the rate of edge of radiotherapy field relapses are low when EUS measurements are included in GTV planning. [11] Further research evaluating the optimal staging strategy with EUS is required.

\section{Staging Techniques}

CT should be performed with intravenous contrast, where appropriate. (Fig.2) The conspicuity of the primary tumour, lymph node involvement and distant metastases is improved.

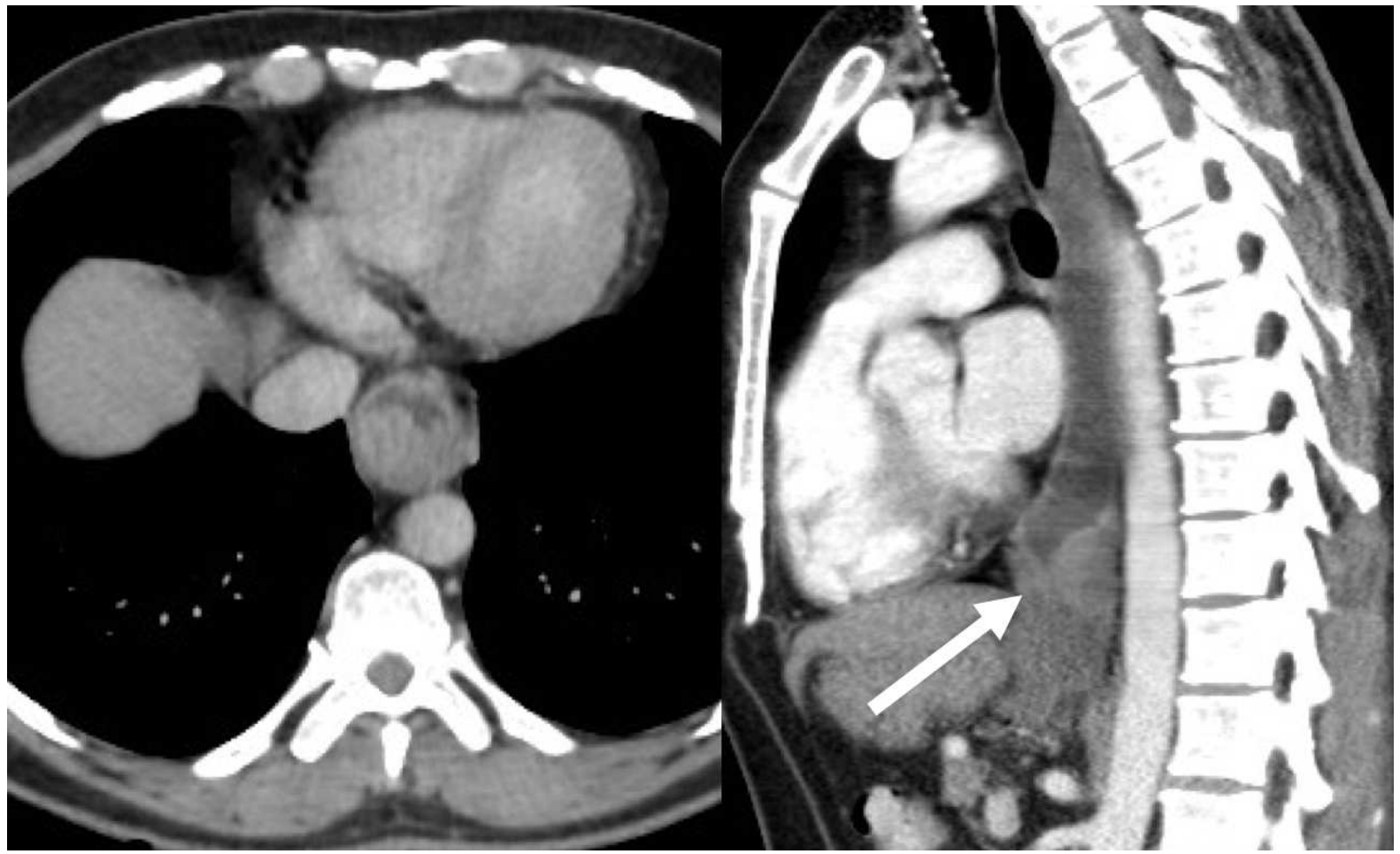


Figure 2. Selected axial and sagittal CT images of a patient with an obstructing distal oesophageal adenocarcinoma (white arrow), staged T3 N2 M0. Residual fluid caused by the luminal narrowing can be seen proximally.

$\mathrm{PET} / \mathrm{CT}$ is routinely used in oesophageal cancer staging pathways worldwide. Strict patient preparation should be adhered to. Patients should be nil-by-mouth for 4-6 hours before scanning and their serum glucose level tested prior to radioisotope injection. [12] All patients should rest before imaging because increased activity in skeletal muscles can increase muscle radioisotope tracer uptake. If the serum glucose level is more than 7 $\mathrm{mmol} / \mathrm{L}$, fluorodeoxyglucose (FDG) uptake in the primary tumour and metastases can be impaired. In addition, metformin can cause increase bowel uptake. [13]

EUS is considered the 'gold standard' imaging technique for assessment of loco-regional stage but is operator dependent. EUS is not without risk; there are potential complications associated with sedation, the risk of oesophageal perforation and failure to cross stenotic tumours. A generally reported failure to cross rate is approximately $30 \%$ but figures as low as $3 \%$ using a slim endoscope have been reported. [14]

Few centres use magnetic resonance imaging (MRI) routinely as a staging investigation and most MRI data in the literature were obtained in a research setting. MRI is technically difficult because the images are prone to movement artefact from the heart, lungs and diaphragm. [15] MRI is potentially valuable when a tumour is impassable with EUS. Results of pathological validation studies have shown promise. [16-18] 
T-stage classifies the depth of tumour invasion by anatomical landmarks and is an important predictor of survival. Five-year overall survival rates approach $82 \%$ when the tumour is limited to the mucosa or submucosa. [19] As described above, the depth of tumour invasion is associated with increasing risk of lymph node metastases. Approximately $74 \%$ of pT3 tumours and $83 \%$ of pT4 tumours have regional lymph node metastases. [20]

An interesting phenomenon that occurs in patients with a tumour situated in a segment of Barrett's oesophagus is duplication of the muscularis mucosae. This has potentially significant implications when staging early superficial tumours and should be considered. [21]

EUS is the most accurate imaging modality for T-staging, with studies finding the accuracy to be more than $80 \%$. $[22,23]$ Due to its superior contrast resolution, the individual layers of the oesophageal wall are well visualised compared to CT and PET. In addition, EUS benefits from 'real-time' imaging to assess for adherence to adjacent structures such as the aorta. (Fig. 3) Encasement of the aorta of more than 180 degrees indicates a highly likelihood of aortic invasion, with more than 270 degrees of encasement almost inevitable for involvement. [24] 


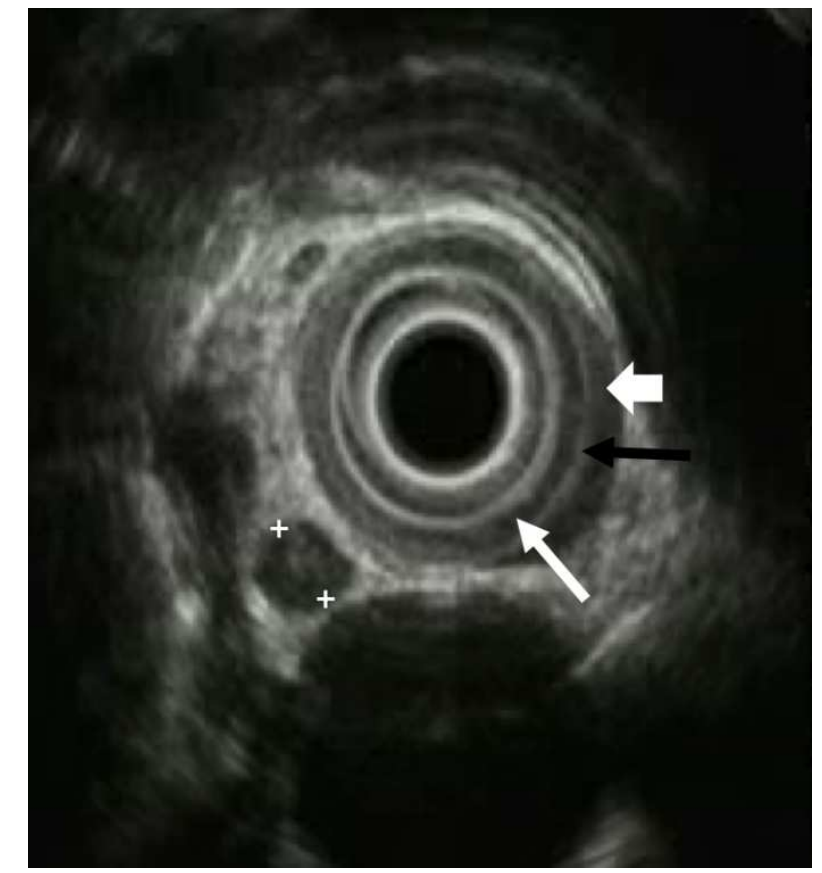

Figure 3. A selected EUS image of an oesophageal tumour with an adjacent lymph node metastasis (white calipers). The inner hypoechoic oesophageal layer (long white arrow) represents the muscularis mucosa, the thin middle hyperechoic layer (black arrow) represents the submucosa and the outer hypoechoic layer (short black arrow) represents the muscularis propria. This tumour was staged T3 because there was invasion through the muscularis propria to the adventitia.

Circumferential involvement of the oesophagus on CT is suggestive of at least T3 disease.

[25] One advantage of CT is the determination of tumour resectability. Identification of fat planes between the tumour and adjacent structures suggests an absence of direct invasion. However, fat planes can be difficult to assess if the tumour abuts an adjacent structure, or when imaged following radiation treatment. The sensitivity and specificity of CT for assessment of resectability is up to $100 \%$ and $80 \%$, respectively. [26] MRI has been reported to have similar accuracy. [27] 
EUS provides excellent contrast resolution and is useful for distinguishing early T1 tumours from more advanced disease, with sensitivity and specificity of $81.6 \%$ and $99.4 \%$, respectively. [22] The sensitivity and specificity of EUS for T1a tumours is $85 \% \& 87 \%$ and $86 \%$ \& $86 \%$ for T1b tumours, respectively. [28]

PET is unlikely to add additional information regarding T-stage. The inherent poor spatial resolution of PET, approximately $5 \mathrm{~mm}$, limits accurate determination of tumour depth. [29] However, an advantage of PET is the quantification of metabolic tumour activity. (Figure 4) Adenocarcinoma and SCC both have a high affinity for FDG, although adenocarcinomas that have high mucinous content, that are poorly differentiated, or have signet-ring subtype can have poor FDG uptake. SCCs tend to have higher FDG uptake than adenocarcinomas. [30] The maximum standardised uptake value $\left(S U V_{\max }\right)$ has been shown to have prognostic significance, although significant results are not always reproduced. [31] 


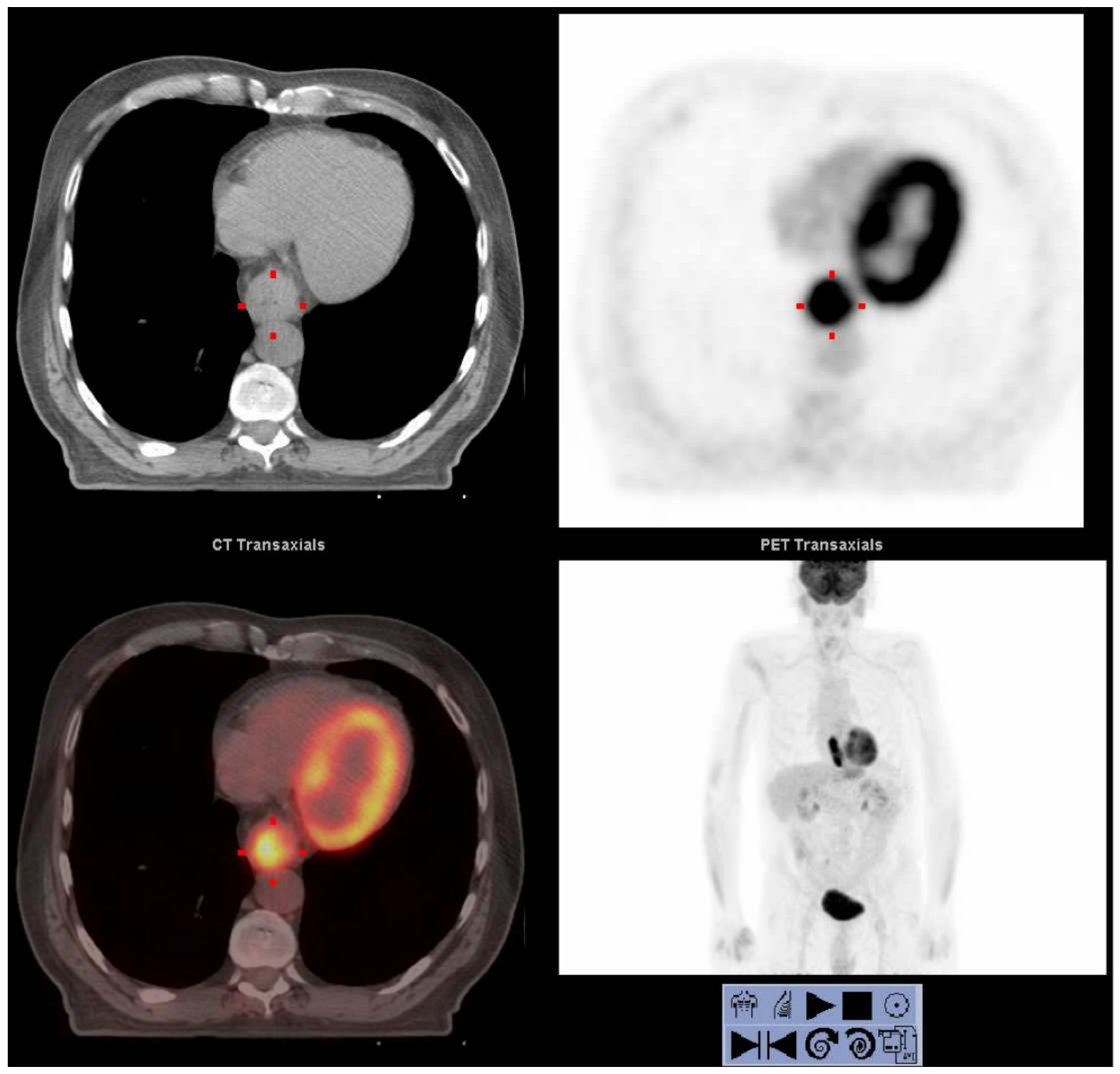

Figure 4. An example of FDG-uptake within a distal oesophageal tumour. The maximum standardised uptake value $\left(S U V_{\max }\right)$ can be quantified and is commonly reported in oesophageal cancer staging. Uptake within the myocardium is commonly seen because of cardiac activity.

Tumour length is an important prognostic factor and has been shown to be independently associated with survival. [32] Pathological correlation of tumour length is challenging because ex-vivo tumours can shrink by up to one third if not fixed and prepared adequately. 
[33] Measurement of tumour and disease length on imaging is important, guiding radiotherapy and surgical decisions, particularly the operation choice based on the location of the predicted proximal and distal margins. Circumferential resection margin involvement (CRM) is now generally considered important for prognosis. [34] Advanced T-stage is independently associated with CRM involvement, with a more than 25 -fold increase in likelihood once the tumour is staged T3 or greater. [35]

\section{$N$-stage}

The TNM classification defines regional lymph nodes as those draining the oesophagus, irrespective of the site of the primary tumour. [36] Coeliac axis and para-oesophageal nodes in the neck are included, but not supra-clavicular lymph nodes. The presence of lymph node metastases is a major prognostic indicator and many studies have shown their significance. [37-40] Patients without lymph node metastases have an overall 5-year survival rate of 70$92 \%$ when treated by surgical resection, but this falls to $18-47 \%$ if metastases are pathologically confirmed. [3]

There are extensive data available on the accuracy of lymph node staging. In general, all staging investigations tend to 'under-stage' lymph node metastases due to their respective limitations. [41] One meta-analysis found the sensitivity of CT, EUS and PET/CT for the detection of regional lymph node metastases was $50 \%, 80 \%$ and $57 \%$, respectively. [7] The specificity was $83 \%, 70 \%$ and $85 \%$, respectively. These figures show that EUS is more sensitive than $\mathrm{CT}$ and $\mathrm{PET}$, therefore correctly excluding more regional lymph node metastases. 
CT relies on anatomical information only and has relatively poor accuracy, with sensitivity as low as $18 \%$. [42] One reason is that CT cannot differentiate between benign and normal sized lymph node metastases. EUS is considered the gold-standard investigation for $\mathrm{N}$ staging. EUS is more sensitive than PET ( $81 \%$ vs $33 \%$ ) but less specific (67\% vs $89 \%$ ). [43] EUS provides the opportunity for fine needle aspiration (FNA), allowing histological confirmation which improves the sensitivity up to 95\%. [22] EUS-FNA of high peri-oesophageal and coeliac axis nodes can also be performed as a 'problem-solver', which adds valuable information when clinicians are deciding whether the patient is suitable for radical therapy.

PET provides descriptive metabolic information about the lymph nodes, however the main limitation of PET is the poor spatial resolution. [44] Detecting small lymph node metastases and differentiating peri-tumoural nodes from the primary tumour can be challenging. A meta-analysis of 12 studies including 490 patients reported an overall sensitivity of $51 \%$ and specificity of $84 \%$ for PET N-staging. [45] A more recent meta-analysis demonstrated that the pooled sensitivity and specificity of PET/CT was $55 \%$ and $76 \%$, respectively. [46]

One study measured the size of the resected lymph nodes and their metastases in patients radiologically staged cNO. The sensitivity of CT, EUS and PET was $39.7 \%, 42.6 \%$ and $35.3 \%$, respectively and the specificity was $77.3 \%, 75.0 \%$ and $90.9 \%$, respectively. [41] The main reason for such poor sensitivity was that $82 \%$ of lymph node metastases measured less than $6 \mathrm{~mm}$ and $44 \%$ less than $2 \mathrm{~mm}$ (classed as micro-metastases), which cannot be visualised on current imaging modalities. Further research is required to improve detection of lymph node metastases in patients with oesophageal cancer. 


\section{M-stage}

The likelihood of distant metastases increases with advanced $\mathrm{T}$ - and $\mathrm{N}$-stage. If unequivocal distant metastatic disease is detected on cross-sectional imaging, the patient receives palliative therapy unless specific oligo-metastatic disease can be resected. The common sites for distant metastatic disease are non-regional lymph nodes, liver, lung and bone.

(Figure 5)

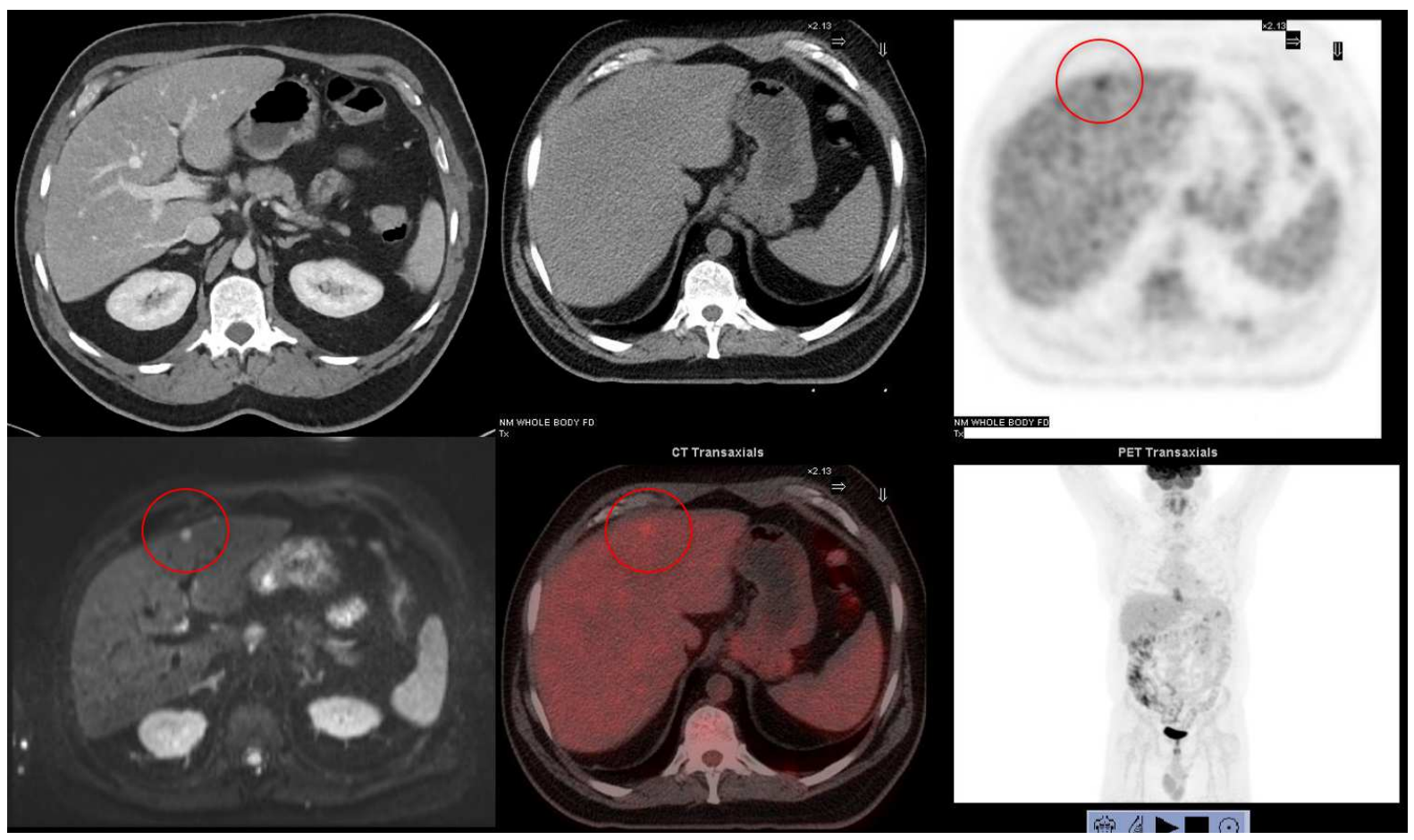

Figure 5. An example of the added value of PET/CT in the oesophageal cancer staging. No focal lesions were visible on $\mathrm{CT}$ (top left). The patient was deemed suitable for radical therapy therefore a PET/CT was performed. This showed a mildly FDG-avid focus in segment 4a of the liver (top right and bottom middle red circles). This lesion was confirmed as a metastasis on MRI diffusion weighted imaging (bottom left). 
The major advancement from the introduction of PET/CT into the oesophageal cancer staging pathway is the superior detection of distant metastases not identified by CT. PET/CT can change management decisions in up to $38 \%$ of patients. [47] PET/CT has a greater sensitivity for distant metastases than CT (71\% versus 52\%, respectively). Specificity for distant metastases is similar (93\% versus 91\%). [7] It is vitally important to detect distant metastatic disease to prevent patients undergoing major intervention when the chances of survival are poor. Quality of life must be considered because surgical patients that die within 2 years of oesophagectomy never fully regain their quality of life. [48]

\section{Re-staging Oesophageal Cancer}

The practice of re-staging is much more varied between institutions with few guidelines available to inform practice. Re-staging with CT and PET/CT is commonplace in the USA, but more restricted in the UK due to the financial constraints of the NHS. Evidence for re-staging with each modality differs but generally the accuracy of repeat investigation and response evaluation is suboptimal. [49] The main questions that re-staging aims to answer are whether the disease is still curable (i.e. to exclude disease progression), how much disease remains (i.e. assessing response) or whether a complete response (confirmed pathologically) has occurred. Re-staging can also be used to assess response during therapy and subsequently guide it, however there is evidence to suggest accuracy rates are suboptimal.

Evaluation of Response 
Whilst response rates to neoadjuvant chemotherapy and chemoradiotherapy vary considerably, a substantial number of patients do not exhibit a meaningful pathological response (indeed, the majority of patients receiving neo-adjuvant chemotherapy). [50] These patients presumably have chemo- or radio-resistant tumours, resulting in a real risk that they develop local or systemic progression during therapy. The gold standard assessment of response is pathological tumour regression grade (TRG), e.g. the Mandard Grade [51], which stratifies patients from complete response (TRG1) to no response (TRG5).

Such disease progression is critical to identify because any attempted surgery will likely be futile, either resulting in an abandoned procedure (due to technical risks during trial dissection), or early 'recurrence' following ostensibly successful resection. This risk has been gradually recognised; in the first generation of neo-adjuvant chemotherapy trials such as the MRC OEO2 trial [52], there were minimal stipulations for staging, and none for restaging. As a result, these trials may have underestimated the survival benefits of neoadjuvant therapy, as they logically must have included patients with incurable disease who still underwent resection. In the OEO5 trial [53], disease progression was demonstrated by CT on an intention to treat basis in $5 \%$ of patients before surgery, whilst in a further $5 \%$ surgery was abandoned due to unsuspected disease progression. In the CROSS trial [54], 4\% of patients randomised to neo-adjuvant chemo-radiotherapy demonstrated diseaseprogression before surgery, with a further $4 \%$ suffering an abandoned resection. These results demonstrate that at least $10 \%$ of patients have disease progression following therapy but more importantly that current imaging strategies are just $50 \%$ sensitive for this. 
The prediction and monitoring of early treatment response using PET/CT is currently of great interest. The MUNICON-1 trial investigated PET uptake pre- and post- neo-adjuvant chemotherapy and found that an SUV $V_{\max }$ reduction of 35\% after 2 weeks predicted response at the end of the 12-week cycle. [55] The trial showed that discontinuation of chemotherapy in metabolic non-responders did not affect their prognosis and prevented further exposure to potential side-effects of treatment. The MUNICON-2 trial investigated the utility of PETguided management, adding radiotherapy to conventional neo-adjuvant chemotherapy in non-responders. [56] PET responders had longer 2-year survival than non-responders (71\% vs $42 \%)$ but this difference did not reach statistical significance $(p=0.10)$.

\section{Imaging Modalities in Re-staging}

As with staging, all three aspects of the TNM classification can be assessed, although these are confounded by the local effects of chemo/radiotherapy. In addition, locally resectable re-staging of $\mathrm{N}$-stage has little effect on operative strategy.

CT is usually performed after neo-adjuvant therapy, traditionally being used to exclude disease progression prior to surgery, rather than assessing response. This is typically done by semi-quantitatively comparing disease volumes using response criteria such as the Response Evaluation Criteria in Solid Tumours (RECIST). [57] However, data suggests that this tool has poor discrimination for classifying response. [58] The use of CT in response assessment is relatively poor, with sensitivity and specificity ranging between $33 \%$ and $55 \%$, and $50 \%$ and $71 \%$, respectively. [59] Whilst not able to precisely stratify response at a microscopic level, CT may provide useful surrogate prognostic information, by identifying down-staging (a reduction in tumour $\mathrm{T}$ - and $\mathrm{N}$-stage). A large study demonstrated that 
down-staging on re-staging investigations provides useful prognostic information. This study found that patients with down-staged tumours after neo-adjuvant chemotherapy experienced improved survival compared with patients without a response $(p<0.001)$, and such down-staging was the strongest independent predictor of survival after adjusting for patient age, tumour grade, pre-treatment stage, lymphovascular invasion, resection margin status and surgical resection type (hazard ratio $0.43 ; 95 \%$ confidence intervals 0.31 0.59). [60]

Although EUS performs well in the initial staging of oesophageal cancer, its value in restaging oesophageal cancer is doubtful. The accuracy of post neo-adjuvant EUS is relatively poor (59\% for both T-stage and N-stage). EUS does not accurately detect down-staging of the tumour, even when a complete pathological response is achieved. [61] Likely reasons for the inaccuracy may be post-treatment inflammation and fibrosis, which distort the architecture of the oesophageal wall, resulting in over-estimation of T-stage even following substantial tumour regression. Accuracy is relatively preserved in patients with minimal response, whilst almost entirely lost in patients with a substantial local response, in whom fibrosis may be indistinguishable from residual tumour. $[62,63]$

Early evidence for MRI, particularly diffusion weighted imaging (DWI) in restaging and treatment response evaluation is promising, although more validation must be performed. Clinical responders have been associated higher apparent diffusion coefficient (ADC) values than non-responders and lower baseline ADC values have been associated with poorer response to chemo-radiotherapy and survival. [64] However, a similar study found an opposing effect, with pathological responders associated with lower baseline ADC values 
and increasing ADC values after neo-adjuvant therapy compared to non-responders. [65] Further research investigating MRI in oesophageal cancer staging, re-staging and treatment planning is required.

\section{PET/CT Response}

The combination of functional and cross-sectional imaging provided by PET/CT has been shown to outperform CT alone. [66] Analogous to RECIST, Positron Emission Tomography Response Criteria in Solid Tumours (PERCIST) uses reduction or progression in tumour avidity to stratify response into complete and partial metabolic response, and stable and progressive metabolic disease. [67] However, sensitivity and specificity for metabolic response is approximately $70 \%$, and hence has yet to show clinical utility. [68] Furthermore, $\mathrm{PET} / \mathrm{CT}$ is unable to reliably exclude or identify $\mathrm{PCR}$ following either neo-adjuvant chemoradiotherapy [66] or chemotherapy. [69] Although not necessarily reliable for individual patients, the majority of patients classified as Mandard TRG 1-3 demonstrate a metabolic response. [70]

Typically, response assessment focusses on the primary tumour. Metabolic response of presumed nodal metastases can also be assessed. Whilst this is usually concordant with the primary tumour, a disparity has been shown in approximately $5 \%$ of patients. [69] In addition, a metabolic nodal response (mNR) has been shown to be a positive prognostic marker, independent of established pathological markers and metabolic response of the primary tumour. [71] It is hypothesised that metabolic tumour response is a surrogate of pathological tumour response (TRG) and $\mathrm{mNR}$ a surrogate of the recently described concept of pathological nodal response [72], although these concepts require validation. Several 
caveats apply to metabolic response of the primary tumour. Determining whether residual avidity is due to tumour necrosis with resultant inflammation, or metabolically active residual tumour is subjective and challenging.

Limitations in response assessment mean that imaging modalities are insufficient for identifying or excluding residual disease in isolation. As a result, studies combining modalities have been attempted, with varying success. One study found little additional benefit of resection when complete response was evident on PET/CT. [73] These data complement the 'watch-and-wait' approach currently being trialled in rectal cancer. [74] Conversely, a combination of EUS and PET/CT following neo-adjuvant chemoradiotherapy was investigated, but was insufficient to accurately select patients for non-operative management. [75] The recent Dutch preSANO study assessed patients following neoadjuvant chemoradiotherapy with endoscopy, biopsies and EUS. [76] Patients with demonstrable residual disease proceeded to surgery, whilst those with possible $\mathrm{pCR}$ were assessed with PET/CT. Significant false negative rates (i.e. missing residual disease) were seen, approximately $10 \%$ for EUS and endoscopic biopsies and a further $15 \%$ for those undergoing PET/CT. This assessment regimen is currently being assessed in the randomised 3 SANO trial.

\section{Insight into evolving imaging techniques}

There are several evolving imaging techniques that, if validated, may improve staging accuracy in oesophageal cancer, enhance the prediction of prognosis, assist in re-staging disease and improve patient outcomes. 
$M R I$

Higher strength MRI scanners have demonstrated excellent T-staging accuracy in studies comparing imaging characteristics with ex-vivo specimens. The accuracy of a 4.7 Tesla MRI scanner was $94 \%$ and replicated with high resolution 1.5 Tesla MRI. $[77,78]$ One hundred percent accuracy has been reported using a 7 Tesla MRI scanner when compared to oesophageal resection specimens [79] but further in-vivo validation is required. If replicated in vivo, MRI may also assist with prediction of CRM involvement, which would be a valuable decision aid when planning the appropriateness and approach of surgery. Whilst wholebody MRI is being trialled in lung and colorectal cancer [80], only pilot data currently exists in oesophageal cancer. [81]

\section{Radiomics}

Radiomics is a current area of great interest. This post-processing technique aims to extract large amounts of additional quantitative data from a selected region of interest within an image. $[82,83]$ These data quantify underlying tumour characteristics by examining the frequency and spatial distribution of individual voxel values. Radiomics can be used to analyse Hounsfield units on $\mathrm{CT}$, signal intensities on MRI and uptake values on PET. Heterogeneity of values within a tumour are thought to reflect variations in angiogenesis, vascularity, hypoxia and necrosis. [84]

Radiomics have been investigated in oesophageal cancer. [85] There have been increasing numbers of significant associations between radiomic parameters/signatures and TNM stage, treatment response and survival found. $[31,86,87]$ However, many of these studies 
include small sample sizes and are likely to report false-positive results. [88] One large, single centre study developed and internally validated a prognostic model including PET radiomics and found that model performance for overall survival improved compared to traditional clinical factors. [89] One critical aspect and potential limitation of radiomics is the reliability and reproducibility of these features. Much radiomics research is now concentrating on defining stable features for future application. [90]

\section{Artificial Intelligence}

Lastly, the application of artificial intelligence techniques in oesophageal cancer is a natural progression of current imaging research. Such applications could include the rapid, automatic detection of a tumour on imaging, the ability to delineate the primary tumour and lymph nodes, disease monitoring during treatment and prediction of response to treatment. The use of deep learning techniques such as convolutional neural networks to predict response to neo-adjuvant chemotherapy have been investigated for PET imaging. [91] There may also be a role for artificial intelligence in treatment planning. 


\section{Summary}

In conclusion, this review presents the latest evidence in oesophageal cancer staging, restaging, response evaluation and novel evolving imaging techniques. Oesophageal cancer staging is complex and treatment decisions are multi-factorial. Accurate staging investigations are vital for management decisions, however, data presented highlight deficiencies, particularly in lymph node staging. In addition, the optimal staging pathway has not yet been agreed. The process of re-staging surgical candidates prior to surgery needs to be standardised and more robust markers of response identified. PET/CT has shown great promise in quantifying treatment response, including early treatment re-assessment, but multi-centre validation studies are lacking. Future oesophageal cancer imaging research should address these areas, aiming to improve staging accuracy, and provide clinicians with a clear evaluation of treatment response or progression. The role of hybrid imaging in treatment planning must also be investigated further. Improved imaging may lead to optimised clinical treatment decisions, which in turn may contribute towards significant advances in the outcomes of patient with oesophageal cancer. 


\section{Practice Points}

- Most patients with oesophageal cancer present with advanced disease

- The risk of lymph node metastases increases with advanced T-stage

- $\quad \mathrm{PET} / \mathrm{CT}$ should be performed before EUS during initial staging

- $\mathrm{PET} / \mathrm{CT}$ has a greater sensitivity for distant metastases than CT alone and changes management in a substantial proportion

- CT should be performed after neo-adjuvant therapy to exclude progression prior to surgery

\section{Research Agenda}

- The optimal oesophageal cancer staging strategy needs to be defined

- Accurate detection of lymph node metastases must be improved

- Improved assessment of treatment response with CT and PET is needed

- The role of imaging to evaluate early response is required

- The role of MRI in staging, response evaluation and treatment planning should be investigated 


\section{References}

1. National Institute for Health and Clinical Excellence. NICE guideline [NG83]:

Oesophago-gastric cancer: assessment and management in adults. 2018 [1st August 2018]; Available from: https://www.nice.org.uk/guidance/ng83.

2. Sobin LH, Gospodarowicz MK, Wittekind CH. UICC TNM Classification of Malignant Tumours. 8th ed: Wiley Blackwell; 2017.

3. Kayani B, Zacharakis E, Ahmed K, Hanna GB. Lymph node metastases and prognosis in oesophageal carcinoma-a systematic review. Eur J Surg Oncol. 2011;37:747-53.

4. Sabik JF, Rice TW, Goldblum JR, Koka A, Kirby TJ, Medendorp SV, et al. Superficial esophageal carcinoma. Ann Thorac Surg. 1995;60:896-901; discussion 2.

5. Allum WH, Blazeby JM, Griffin SM, Cunningham D, Jankowski JA, Wong R. Guidelines for the management of oesophageal and gastric cancer. Gut. 2011;60:1449-72.

6. Schreurs LM, Janssens AC, Groen H, Fockens $P$, van Dullemen HM, van Berge Henegouwen MI, et al. Value of EUS in Determining Curative Resectability in Reference to CT and FDG-PET: The Optimal Sequence in Preoperative Staging of Esophageal Cancer? Ann Surg Oncol. 2011.

7. van Vliet EP, Heijenbrok-Kal MH, Hunink MG, Kuipers EJ, Siersema PD. Staging investigations for oesophageal cancer: a meta-analysis. Br J Cancer. 2008;98:547-57.

8. Findlay JM, Bradley KM, Maile EJ, Braden B, Maw J, Phillips-Hughes J, et al. Pragmatic staging of oesophageal cancer using decision theory involving selective endoscopic ultrasonography, PET and laparoscopy. Br J Surg. 2015;102:1488-99.

9. Hulshoff JB, Mul VE, de Boer HE, Noordzij W, Korteweg T, van Dullemen HM, et al. Impact of Endoscopic Ultrasonography on 18F-FDG-PET/CT Upfront Towards Patient Specific Esophageal Cancer Treatment. Ann Surg Oncol. 2017.

10. Foley KG, Morgan C, Roberts SA, Crosby T. Impact of Positron Emission Tomography and Endoscopic Ultrasound Length of Disease Difference on Treatment Planning in Patients with Oesophageal Cancer. Clin Oncol (R Coll Radiol). 2017;29:760-6.

11. Button MR, Morgan CA, Croydon ES, Roberts SA, Crosby TD. Study to determine adequate margins in radiotherapy planning for esophageal carcinoma by detailing patterns of recurrence after definitive chemoradiotherapy. Int J Radiat Oncol Biol Phys. 2009;73:81823.

12. Kinahan PE, Fletcher JW. Positron emission tomography-computed tomography standardized uptake values in clinical practice and assessing response to therapy. Semin Ultrasound CT MR. 2010;31:496-505.

13. Gontier E, Fourme E, Wartski M, Blondet C, Bonardel G, Le Stanc E, et al. High and typical 18F-FDG bowel uptake in patients treated with metformin. Eur J Nucl Med Mol Imaging. 2008;35:95-9.

14. Morgan MA, Twine CP, Lewis WG, Lambe R, Oliphant HE, Robinson M, et al. Prognostic significance of failure to cross esophageal tumors by endoluminal ultrasound. Dis Esophagus. 2008;21:508-13.

15. Riddell AM, Richardson C, Scurr E, Brown G. The development and optimization of high spatial resolution MRI for imaging the oesophagus using an external surface coil. Br J Radiol. 2006;79:873-9.

16. Giganti F, Ambrosi A, Petrone MC, Canevari C, Chiari D, Salerno A, et al. Prospective comparison of MR with diffusion-weighted imaging, endoscopic ultrasound, MDCT and 
positron emission tomography-CT in the pre-operative staging of oesophageal cancer: results from a pilot study. Br J Radiol. 2016;89:20160087.

17. Li QW, Qiu B, Wang B, Wang DL, Yin SH, Yang H, et al. Prediction of pathologic responders to neoadjuvant chemoradiotherapy by diffusion-weighted magnetic resonance imaging in locally advanced esophageal squamous cell carcinoma: a prospective study. Dis Esophagus. 2018;31.

18. Qu J, Zhang H, Wang Z, Zhang F, Liu H, Ding Z, et al. Comparison between freebreathing radial VIBE on 3-T MRI and endoscopic ultrasound for preoperative T staging of resectable oesophageal cancer, with histopathological correlation. Eur Radiol. 2018;28:7807.

19. Paraf F, Flejou JF, Pignon JP, Fekete F, Potet F. Surgical pathology of adenocarcinoma arising in Barrett's esophagus. Analysis of 67 cases. Am J Surg Pathol. 1995;19:183-91. 20. Ide H, Nakamura T, Hayashi K, Endo T, Kobayashi A, Eguchi R, et al. Esophageal squamous cell carcinoma: pathology and prognosis. World J Surg. 1994;18:321-30.

21. Abraham SC, Krasinskas AM, Correa AM, Hofstetter WL, Ajani JA, Swisher SG, et al. Duplication of the muscularis mucosae in Barrett esophagus: an underrecognized feature and its implication for staging of adenocarcinoma. Am J Surg Pathol. 2007;31:1719-25.

22. Puli SR, Reddy JB, Bechtold ML, Antillon D, Ibdah JA, Antillon MR. Staging accuracy of esophageal cancer by endoscopic ultrasound: a meta-analysis and systematic review. World J Gastroenterol. 2008;14:1479-90.

23. Rosch T. Endosonographic staging of esophageal cancer: a review of literature results. Gastrointest Endosc Clin N Am. 1995;5:537-47.

24. Herman SJ, Winton TL, Weisbrod GL, Towers MJ, Mentzer SJ. Mediastinal invasion by bronchogenic carcinoma: CT signs. Radiology. 1994;190:841-6.

25. Li H, Chen TW, Zhang XM, Li ZL, Chen XL, Tang HJ, et al. Computed tomography scan as a tool to predict tumor T category in resectable esophageal squamous cell carcinoma. Ann Thorac Surg. 2013;95:1749-55.

26. Takashima S, Takeuchi N, Shiozaki H, Kobayashi K, Morimoto S, Ikezoe J, et al. Carcinoma of the esophagus: CT vs MR imaging in determining resectability. AJR Am J Roentgenol. 1991;156:297-302.

27. van Rossum PS, van Hillegersberg R, Lever FM, Lips IM, van Lier AL, Meijer GJ, et al. Imaging strategies in the management of oesophageal cancer: what's the role of MRI? Eur Radiol. 2013;23:1753-65.

28. Thosani N, Singh H, Kapadia A, Ochi N, Lee JH, Ajani J, et al. Diagnostic accuracy of EUS in differentiating mucosal versus submucosal invasion of superficial esophageal cancers: a systematic review and meta-analysis. Gastrointest Endosc. 2012;75:242-53.

29. Levin CS. Promising new photon detection concepts for high-resolution clinical and preclinical PET. J Nucl Med. 2012;53:167-70.

30. de Geus-Oei LF, van Krieken JH, Aliredjo RP, Krabbe PF, Frielink C, Verhagen AF, et al. Biological correlates of FDG uptake in non-small cell lung cancer. Lung Cancer. 2007;55:7987.

31. Hatt M, Majdoub M, Vallieres M, Tixier F, Le Rest CC, Groheux D, et al. 18F-FDG PET Uptake Characterization Through Texture Analysis: Investigating the Complementary Nature of Heterogeneity and Functional Tumor Volume in a Multi-Cancer Site Patient Cohort. J Nucl Med. 2015;56:38-44. 
32. Yendamuri S, Swisher SG, Correa AM, Hofstetter W, Ajani JA, Francis A, et al. Esophageal tumor length is independently associated with long-term survival. Cancer. 2009;115:508-16.

33. Siu KF, Cheung HC, Wong J. Shrinkage of the esophagus after resection for carcinoma. Ann Surg. 1986;203:173-6.

34. Chan DS, Reid TD, Howell I, Lewis WG. Systematic review and meta-analysis of the influence of circumferential resection margin involvement on survival in patients with operable oesophageal cancer. Br J Surg. 2013;100:456-64.

35. Reid TD, Chan DS, Roberts SA, Crosby TD, Williams GT, Lewis WG. Prognostic significance of circumferential resection margin involvement following oesophagectomy for cancer and the predictive role of endoluminal ultrasonography. Br J Cancer. 2012;107:192531.

36. Sobin LH, Gospodarowicz MK, Wittekind CH. UICC TNM Classification of Malignant Tumours. 7th ed. New York: Wiley; 2009.

37. Rizk N, Venkatraman E, Park B, Flores R, Bains MS, Rusch V. The prognostic importance of the number of involved lymph nodes in esophageal cancer: implications for revisions of the American Joint Committee on Cancer staging system. J Thorac Cardiovasc Surg. 2006;132:1374-81.

38. O'Riordan JM, Rowley S, Murphy JO, Ravi N, Byrne PJ, Reynolds JV. Impact of solitary involved lymph node on outcome in localized cancer of the esophagus and esophagogastric junction. J Gastrointest Surg. 2007;11:493-9.

39. Mariette C, Piessen G, Briez N, Triboulet JP. The number of metastatic lymph nodes and the ratio between metastatic and examined lymph nodes are independent prognostic factors in esophageal cancer regardless of neoadjuvant chemoradiation or lymphadenectomy extent. Ann Surg. 2008;247:365-71.

40. Twine CP, Roberts SA, Rawlinson CE, Davies L, Escofet X, Dave BV, et al. Prognostic significance of the endoscopic ultrasound defined lymph node metastasis count in esophageal cancer. Dis Esophagus. 2010;23:652-9.

41. Foley KG, Christian A, Fielding P, Lewis WG, Roberts SA. Accuracy of contemporary oesophageal cancer lymph node staging with radiological-pathological correlation. Clin Radiol. 2017;72:e691-e7.

42. Choi JY, Lee KH, Shim YM, Lee KS, Kim JJ, Kim SE, et al. Improved detection of individual nodal involvement in squamous cell carcinoma of the esophagus by FDG PET. J Nucl Med. 2000;41:808-15.

43. Flamen P, Lerut A, Van Cutsem E, De Wever W, Peeters M, Stroobants S, et al. Utility of positron emission tomography for the staging of patients with potentially operable esophageal carcinoma. J Clin Oncol. 2000;18:3202-10.

44. Kapoor V, McCook BM, Torok FS. An introduction to PET-CT imaging. Radiographics. 2004;24:523-43.

45. Flanagan FL, Dehdashti F, Siegel BA, Trask DD, Sundaresan SR, Patterson GA, et al. Staging of esophageal cancer with F-18-fluorodeoxyglucose positron emission tomography. American Journal of Roentgenology. 1997;168:417-24.

46. Shi W, Wang W, Wang J, Cheng H, Huo X. Meta-analysis of 18FDG PET-CT for nodal staging in patients with esophageal cancer. Surg Oncol. 2013;22:112-6.

47. Blencowe NS, Whistance RN, Strong S, Hotton EJ, Ganesh S, Roach H, et al. Evaluating the role of fluorodeoxyglucose positron emission tomography-computed 
tomography in multi-disciplinary team recommendations for oesophago-gastric cancer. $\mathrm{Br} \mathrm{J}$ Cancer. 2013;109:1445-50.

48. Blazeby JM, Farndon JR, Donovan J, Alderson D. A prospective longitudinal study examining the quality of life of patients with esophageal carcinoma. Cancer. 2000;88:17817.

49. Yip C, Cook GJ, Landau DB, Davies A, Goh V. Performance of different imaging modalities in assessment of response to neoadjuvant therapy in primary esophageal cancer. Dis Esophagus. 2016;29:116-30.

50. Sjoquist KM, Burmeister BH, Smithers BM, Zalcberg JR, Simes RJ, Barbour A, et al. Survival after neoadjuvant chemotherapy or chemoradiotherapy for resectable oesophageal carcinoma: an updated meta-analysis. Lancet Oncol. 2011;12:681-92.

51. Mandard AM, Dalibard F, Mandard JC, Marnay J, Henry-Amar M, Petiot JF, et al. Pathologic assessment of tumor regression after preoperative chemoradiotherapy of esophageal carcinoma. Clinicopathologic correlations. Cancer. 1994;73:2680-6.

52. Medical Research Council Oesophageal Cancer Working Group. Surgical resection with or without preoperative chemotherapy in oesophageal cancer: a randomised controlled trial. Lancet. 2002;359:1727-33.

53. Alderson D, Cunningham D, Nankivell M, Blazeby JM, Griffin SM, Crellin A, et al. Neoadjuvant cisplatin and fluorouracil versus epirubicin, cisplatin, and capecitabine followed by resection in patients with oesophageal adenocarcinoma (UK MRC OE05): an open-label, randomised phase 3 trial. Lancet Oncol. 2017;18:1249-60.

54. van Hagen P, Hulshof MCCM, van Lanschot JJB, Steyerberg EW, van Berge Henegouwen MI, Wijnhoven BPL, et al. Preoperative chemoradiotherapy for esophageal or junctional cancer. N Engl J Med. 2012;366:2074-84.

55. Javeri $\mathrm{H}$, Xiao L, Rohren $\mathrm{E}$, Komaki R, Hofstetter $\mathrm{W}$, Lee $\mathrm{JH}$, et al. Influence of the baseline 18F-fluoro-2-deoxy-D-glucose positron emission tomography results on survival and pathologic response in patients with gastroesophageal cancer undergoing chemoradiation. Cancer. 2009;115:624-30.

56. zum Buschenfelde CM, Herrmann K, Schuster T, Geinitz H, Langer R, Becker K, et al. (18)F-FDG PET-guided salvage neoadjuvant radiochemotherapy of adenocarcinoma of the esophagogastric junction: the MUNICON II trial. J Nucl Med. 2011;52:1189-96.

57. Eisenhauer EA, Therasse P, Bogaerts J, Schwartz LH, Sargent D, Ford R, et al. New response evaluation criteria in solid tumours: revised RECIST guideline (version 1.1). Eur J Cancer. 2009;45:228-47.

58. Kurokawa Y, Shibata T, Ando N, Seki S, Mukaida H, Fukuda H. Which is the optimal response criteria for evaluating preoperative treatment in esophageal cancer: RECIST or histology? Ann Surg Oncol. 2013;20:3009-14.

59. Isenberg G, Chak A, Canto MI, Levitan N, Clayman J, Pollack BJ, et al. Endoscopic ultrasound in restaging of esophageal cancer after neoadjuvant chemoradiation.

Gastrointest Endosc. 1998;48:158-63.

60. Davies AR, Gossage JA, Zylstra J, Mattsson F, Lagergren J, Maisey N, et al. Tumor stage after neoadjuvant chemotherapy determines survival after surgery for adenocarcinoma of the esophagus and esophagogastric junction. J Clin Oncol. 2014;32:2983-90.

61. Bowrey DJ, Clark GW, Roberts SA, Hawthorne AB, Maughan TS, Williams GT, et al. Serial endoscopic ultrasound in the assessment of response to chemoradiotherapy for carcinoma of the esophagus. J Gastrointest Surg. 1999;3:462-7. 
62. Beseth BD, Bedford R, Isacoff WH, Holmes EC, Cameron RB. Endoscopic ultrasound does not accurately assess pathologic stage of esophageal cancer after neoadjuvant chemoradiotherapy. Am Surg. 2000;66:827-31.

63. Willis J, Cooper GS, Isenberg G, Sivak MV, Jr., Levitan N, Clayman J, et al. Correlation of EUS measurement with pathologic assessment of neoadjuvant therapy response in esophageal carcinoma. Gastrointest Endosc. 2002;55:655-61.

64. Aoyagi T, Shuto K, Okazumi S, Shimada H, Kazama T, Matsubara H. Apparent diffusion coefficient values measured by diffusion-weighted imaging predict chemoradiotherapeutic effect for advanced esophageal cancer. Dig Surg. 2011;28:252-7. 65. De Cobelli F, Giganti F, Orsenigo E, Cellina M, Esposito A, Agostini G, et al. Apparent diffusion coefficient modifications in assessing gastro-oesophageal cancer response to neoadjuvant treatment: comparison with tumour regression grade at histology. Eur Radiol. 2013;23:2165-74.

66. Swisher SG, Maish M, Erasmus JJ, Correa AM, Ajani JA, Bresalier R, et al. Utility of PET, CT, and EUS to identify pathologic responders in esophageal cancer. Ann Thorac Surg. 2004;78:1152-60; discussion -60.

67. Wahl RL, Jacene $H$, Kasamon $\mathrm{Y}$, Lodge MA. From RECIST to PERCIST: Evolving Considerations for PET Response Criteria in Solid Tumours. J Nucl Med. 2009;50:122S-50S. 68. Odawara S, Kitajima K, Katsuura T, Kurahashi Y, Shinohara H, Yamakado K. Tumor response to neoadjuvant chemotherapy in patients with esophageal cancer assessed with CT and FDG-PET/CT - RECIST 1.1 vs. PERCIST 1.0. Eur J Radiol. 2018;101:65-71.

69. Findlay JM, Bradley KM, Wang LM, Franklin JM, Teoh EJ, Gleeson FV, et al. Predicting Pathologic Response of Esophageal Cancer to Neoadjuvant Chemotherapy: The Implications of Metabolic Nodal Response for Personalized Therapy. J Nucl Med. 2017;58:266-75.

70. Kukar M, Alnaji RM, Jabi F, Platz TA, Attwood K, Nava H, et al. Role of Repeat 18FFluorodeoxyglucose Positron Emission Tomography Examination in Predicting Pathologic Response Following Neoadjuvant Chemoradiotherapy for Esophageal Adenocarcinoma. JAMA surgery. 2015;150:555-62.

71. Findlay JM, Bradley KM, Wang LM, Franklin JM, Teoh EJ, Gleeson FV, et al. Metabolic nodal response as a prognostic marker after neoadjuvant therapy for oesophageal cancer. Br J Surg. 2017;104:408-17.

72. Davies AR, Myoteri D, Zylstra J, Baker CR, Wulaningsih W, Van Hemelrijck M, et al. Lymph node regression and survival following neoadjuvant chemotherapy in oesophageal adenocarcinoma. Br J Surg. 2018.

73. Monjazeb AM, Riedlinger G, Aklilu M, Geisinger KR, Mishra G, Isom S, et al.

Outcomes of patients with esophageal cancer staged with 18F-fluorodeoxyglucose positron emission tomography (FDG-PET): can postchemoradiotherapy FDG-PET predict the utility of resection? J Clin Oncol. 2010;28:4714-21.

74. van der Valk MJM, Hilling DE, Bastiaannet E, Meershoek-Klein Kranenbarg E, Beets GL, Figueiredo NL, et al. Long-term outcomes of clinical complete responders after neoadjuvant treatment for rectal cancer in the International Watch \& Wait Database (IWWD): an international multicentre registry study. Lancet. 2018;391:2537-45.

75. Heneghan HM, Donohoe C, Elliot J, Ahmed Z, Malik V, Ravi N, et al. Can CT-PET and Endoscopic Assessment Post-Neoadjuvant Chemoradiotherapy Predict Residual Disease in Esophageal Cancer? Ann Surg. 2016;264:831-8.

76. Noordman BJ, Spaander MCW, Valkema R, Wijnhoven BPL, van Berge Henegouwen $\mathrm{MI}$, Shapiro J, et al. Detection of residual disease after neoadjuvant chemoradiotherapy for 
oesophageal cancer (preSANO): a prospective multicentre, diagnostic cohort study. Lancet Oncol. 2018;19:965-74.

77. Yamada I, Murata Y, Izumi Y, Kawano T, Endo M, Kuroiwa T, et al. Staging of esophageal carcinoma in vitro with 4.7-T MR imaging. Radiology. 1997;204:521-6.

78. Yamada I, Izumi Y, Kawano T, Yoshino N, Tetsumura A, Ohashi K, et al. Superficial esophageal carcinoma: an in vitro study of high-resolution MR imaging at 1.5T. J Magn Reson Imaging. 2001;13:225-31.

79. Yamada I, Hikishima K, Miyasaka N, Kawano T, Tokairin Y, Ito E, et al. Esophageal carcinoma: ex vivo evaluation with diffusion-tensor MR imaging and tractography at $7 \mathrm{~T}$. Radiology. 2014;272:164-73.

80. Taylor SA, Mallett S, Miles A, Beare S, Bhatnagar G, Bridgewater J, et al. Streamlining staging of lung and colorectal cancer with whole body MRI; study protocols for two multicentre, non-randomised, single-arm, prospective diagnostic accuracy studies (Streamline C and Streamline L). BMC Cancer. 2017;17:299.

81. Malik V, Harmon M, Johnston C, Fagan AJ, Claxton Z, Ravi N, et al. Whole Body MRI in the Staging of Esophageal Cancer--A Prospective Comparison with Whole Body 18F-FDG PET-CT. Dig Surg. 2015;32:397-408.

82. Lambin P, Rios-Velazquez E, Leijenaar R, Carvalho S, van Stiphout RG, Granton P, et al. Radiomics: extracting more information from medical images using advanced feature analysis. Eur J Cancer. 2012;48:441-6.

83. Gillies RJ, Kinahan PE, Hricak H. Radiomics: Images Are More than Pictures, They Are Data. Radiology. 2016;278:563-77.

84. Henriksson E, Kjellen E, Wahlberg P, Ohlsson T, Wennerberg J, Brun E. 2-Deoxy-2[18F] fluoro-D-glucose uptake and correlation to intratumoral heterogeneity. Anticancer Res. 2007;27:2155-9.

85. van Rossum PS, Xu C, Fried DV, Goense L, Court LE, Lin SH. The emerging field of radiomics in esophageal cancer: current evidence and future potential. Transl Cancer Res. 2016;5:410-23.

86. Dong X, Xing L, Wu P, Fu Z, Wan H, Li D, et al. Three-dimensional positron emission tomography image texture analysis of esophageal squamous cell carcinoma: relationship between tumor 18F-fluorodeoxyglucose uptake heterogeneity, maximum standardized uptake value, and tumor stage. Nucl Med Commun. 2013;34:40-6.

87. Yip C, Landau D, Kozarski R, Ganeshan B, Thomas R, Michaelidou A, et al. Primary esophageal cancer: heterogeneity as potential prognostic biomarker in patients treated with definitive chemotherapy and radiation therapy. Radiology. 2014;270:141-8.

88. Chalkidou A, O'Doherty MJ, Marsden PK. False Discovery Rates in PET and CT Studies with Texture Features: A Systematic Review. PLoS One. 2015;10:e0124165.

89. Foley KG, Hills RK, Berthon B, Marshall C, Parkinson C, Lewis WG, et al. Development and validation of a prognostic model incorporating texture analysis derived from standardised segmentation of PET in patients with oesophageal cancer. Eur Radiol. 2018;28:428-36.

90. Zwanenburg A, International Biomarker Standardisation Initiative (IBSI). EP-1677. Multicentre Initiative for Standardisation of Image Biomarkers. Radiother Oncol. 2017;127 Suppl 1:S543-S4.

91. Ypsilantis PP, Siddique M, Sohn HM, Davies A, Cook G, Goh V, et al. Predicting Response to Neoadjuvant Chemotherapy with PET Imaging Using Convolutional Neural Networks. PLoS One. 2015;10:e0137036. 
